\title{
Mechanics of pH-Responsive Hydrogel Capsules
}

\author{
James P. Best, ${ }^{\dagger}$ Martin P. Neubauer, ${ }^{\ddagger}$ Sameen Javed, ${ }^{\dagger}$ Henk H. Dam, ${ }^{\dagger}$ Andreas Fery, ${ }^{*}{ }^{\ddagger}$ and Frank \\ Caruso*,†
}

†Department of Chemical and Biomolecular Engineering, The University of Melbourne, Parkville, Victoria 3010, Australia, and ${ }^{\ddagger}$ Physical Chemistry II, Bayreuth University, 95447 Bayreuth, Germany

KEYWORDS. Hydrogel, capsule, polymer film, atomic force microscopy, colloidal probe, mechanical properties

\begin{abstract}
While soft hydrogel nano- and microstructures hold great potential for therapeutic treatments and in vivo applications, their nanomechanical characterization remains a challenge. In this article, soft, single-component supported hydrogel films were fabricated using pendant-thiol modified poly(methacrylic acid) (PMAsH). The influence of hydrogel architecture on deformation properties was studied by fabricating films on particle supports and producing free-standing capsules. The influence of the degree of thiol-based crosslinking on the mechanical properties of the soft hydrogel systems (core-shell and capsules) was studied using a colloidal-probe (CP) AFM technique. It was found that film mechanical properties, stability, and capsule swelling could be finely tuned by controlling the extent of poly(methacrylic acid) thiol modification. Furthermore, switching the pH from 7.4 to 4.0 led to film densification due to increased hydrogen bonding. Hydrogel capsule systems were found to have stiffness values ranging from 0.9 to $16.9 \mathrm{mN} \mathrm{m}^{-1}$ over a thiol modification range of 5 to $20 \mathrm{~mol} \%$. These values are significantly greater than those for previously reported PMAsH planar films of 0.7 to $5.7 \mathrm{mN} \mathrm{m}^{-1}$ over the same thiol modification range (Best et al., Soft Matter 2013, 9, 4580-4584). Films on particle substrates had comparable mechanical properties to planar films, demonstrating that while substrate geometry has a negligible effect, membrane and tension effects may play an important role in capsule force resistance. Further, when transitioning from solidsupported films to free-standing capsules, simple predictions of shell stiffness based on modulus changes found for solid supported results are not valid. Rather, additional effects like diameter increases (geometrical changes) as well as tension buildup need to be taken into account. These results are important for research related to the characterization of soft hydrogel materials and control over their mechanical properties.
\end{abstract}

\section{INTRODUCTION}

Soft nanostructured planar and particle hydrogel systems are promising for biomedical applications due to their low cytotoxicity and tunable hydration and rigidity. ${ }^{1-4}$ Increasingly, research is focusing on the influence of advanced material physicochemical properties on cellular and biological interactions. ${ }^{5,6}$ While this is primarily directed to the delivery of therapeutics for in vivo systems, its scope extends as far as the understanding of basic cellular function and activity. In a recent example, Giasson and coworkers investigated the effect of hydrogel nanoparticle uptake by RAW 264.7 murine macrophage cells. ${ }^{7}$ The mechanical properties of the hydrogel particles were controlled through the crosslinking degree, and it was found that both the rate and endocytosis mechanism utilized by the cells were governed by the rigidity of the particles studied. In addition to particle systems, the influence of hydrogel film mechanical properties on cellular adhesion, migration, proliferation, and growth has also been studied. ${ }^{8}$ We recently reported that by tuning the stiffness of the thiolated hydrogel films presented here, cervical cancer cell adhesion was able to be mediated. ${ }^{9}$ Such studies are likely to influence the design of future hydrogel materials for tissue engineering and targeted drug delivery applications. However, our current understanding of the properties of free-standing capsule systems is largely based on studies extrapolated from films assembled on planar supports.

Atomic force microscopy (AFM) force spectroscopy is a useful technique for the mechanical analysis of soft nanostructured materials. AFM has been used for the characterization of planar thin-films, ${ }^{10-12}$ particles, ${ }^{7,13}$ and hollow polyelectrolyte capsules ${ }^{14-16}$ with great precision. One of the advantages of such a technique is that single capsules (or particles) can be independently measured, offering considerable mechanical information. However, collecting sufficient data for a reasonable statistical analysis can be time consuming. Furthering the single particle measurement technique, in 1991 Ducker et al. developed a colloidal probe method for higher sensitivity force measurements. ${ }^{17}$ Useful for especially soft materials, a colloidal probe (CP) is attached to the end of a cantilever to increase 
the interaction area when compared to a sharp tip probe. ${ }^{18}$ This also offers the advantage of having a well-defined interaction geometry, tailored surface roughness and chemistry, and reduced localized axial strain during measurement. ${ }^{18,19}$ The latter is particularly important, as for large applied axial strains, material nonlinearity can be observed. ${ }^{19}$ The CP-AFM technique has been used for the characterization and Young's modulus $\left(E_{Y}\right)$ determination of polyelectrolyte multilayer films ${ }^{20}$ and capsules, ${ }^{21,22}$ and has been successfully utilized to investigate large hydrogel capsules undergoing $\mathrm{pH}$ cycling in buffer under low applied forces. ${ }^{23}$ However, apart from the latter study, the tuning and analysis of extremely soft free-standing hydrogel particles has received sparse attention. It is also important to note that while the swelling behavior of planar film and capsule poly(methacrylic acid) (PMA) systems has been investigated, ${ }^{24,25}$ no study has compared the mechanical properties of materials on various substrates with different dimensionalities.

Thiol-modified poly(methacrylic acid) ( $\left.\mathrm{PMA}_{\mathrm{SH}}\right)$ can be used to form tunable hydrogel systems that have been the focus of recent studies. ${ }^{26-28}$ Single-component films and hydrogel capsules (HCs) can be formed using the layer-bylayer (LbL) assembly technique, through cooperative hydrogen bonding interactions between PMA and poly $(N$ vinyl pyrrolidone) (PVPON). PMAsH can be then crosslinked through oxidation of the pendant thiol groups into disulfide linkages, and the PVPON removed at physiological $\mathrm{pH}$ by disrupting the hydrogen bonding network. As the pKa of PMA is 6-7, carboxylic acid groups are deprotonated at $\mathrm{pH} 7.4$, leading to intermolecular charge repulsion and capsule swelling. Cross-linked PMAsH systems, however, are able to form highly hydrated and stable networks at high $\mathrm{pH}$. In addition, the disulfide cross-links between adjacent polymers may be cleaved under reducing conditions, such as for intracellular environments, which may facilitate material degradation and cargo release in vivo. Specifically, PMAsн systems have been shown to be degradable in simulated intracellular conditions ${ }^{29}$ while demonstrating stability at bloodstream $\mathrm{pH}^{30} \mathrm{PMA}_{\mathrm{sH}}$ capsules can be antibody-functionalized for enhanced cell binding and association, ${ }^{31}$ and can also encapsulate proteins, ${ }^{30}$ oligonucleotides, ${ }^{32}$ and double-stranded linear DNA or plasmid DNA. ${ }^{33}$ These characteristics demonstrate the considerable promise of PMAsH hydrogel materials for future therapeutic treatments.

In this article, we quantify the extent of 2-(2pyridinyldithio)ethaneamine (PDA) modification of PMA (PMA-PDA), which allows for disulfide cross-link formation, for four different PDA target percentages using UV-Vis spectrophotometry. We then examine the structure and LbL assembly of the constituent PMAsH polymers on particle substrates, and quantify the mechanical properties. In particular, we investigate how the degree of crosslinking in these systems affects HC film stability and system stiffness, using CP-AFM. This approach allows for the investigation of free-standing shell mechanics in hollow capsule systems, which can then be compared to soft hydrogel planar films previously reported. ${ }^{9}$ This provides insights into the effects of hydrogel architecture on mechanical resistances at deformations on the nanoscale.

\section{EXPERIMENTAL METHODS}

Materials. The $\mathrm{pH}$ of all solutions was measured with a Mettler-Toledo MP220 pH meter. High purity (Milli-Q) water with a resistivity of $>18 \mathrm{M} \Omega \cdot \mathrm{cm}^{-1}$ was obtained from an inline Millipore RiOs/Origin water purification system. Dialysis tubing (68035-35FT) with a MWCO of 3500 Da was obtained from Thermo Scientific. $\mathrm{SiO}_{2}$ particles $(1.11 \pm$ $0.05 \mu \mathrm{m}$ diameter) were purchased from Microparticles $\mathrm{GmbH}$ (Berlin, Germany) as a $50 \mathrm{~g} \mathrm{~L}^{-1}$ suspension. Poly(methacrylic acid, sodium salt), (PMA-Na, $M_{\mathrm{w}}=15000$ $\mathrm{g} \mathrm{mol}^{-1}$ ) was purchased from Polysciences, while PVPON

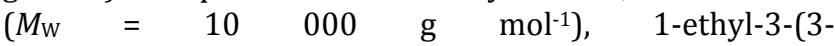
dimethylaminopropyl)carbodiimide (EDC), dithiothreitol (DTT), polyethyleneimine (PEI, $25000 \mathrm{~g} \mathrm{~mol}^{-1}$ ), phosphate buffered saline (PBS), 3-( $N$-morpholino)propanesulfonic acid (MOPS), 2-( $N$-morpholino)ethanesulfonic acid (MES), sodium acetate (NaOAc), $N$-chloro- $p$-toluenesulfonamide sodium salt (CaT), hydrofluoric acid (HF), and PDA were obtained from Sigma-Aldrich. All chemicals were used as received. Mica (grade V-4) substrates of $22 \mathrm{~mm}$ diameter were sourced from SPI Chem (USA). PMA-PDA was synthesized and characterized using nuclear magnetic resonance spectroscopy ( ${ }^{1} \mathrm{H}-\mathrm{NMR}, 500 \mathrm{MHz}$ Varian INOVA system at $25^{\circ} \mathrm{C}$ ) as previously reported. ${ }^{9}$

Prior to LbL assembly, the PMA-PDA precursor was reduced to $\mathrm{PMA}_{\mathrm{SH}}$ through cleavage of the PDA group. The reduction required a $0.5 \mathrm{M}$ DTT solution in $50 \mathrm{mM}$ MOPS buffer ( $\mathrm{pH}$ 8) for $30 \mathrm{~min}$ at $37{ }^{\circ} \mathrm{C}$ with constant agitation. The resultant $\mathrm{PMA}_{\mathrm{SH}}$ solution was then diluted with 100 $\mathrm{mM} \mathrm{NaOAc} \mathrm{buffer} \mathrm{(pH} \mathrm{4)} \mathrm{to} 0.5 \mathrm{mg} \mathrm{mL}^{-1}$ for planar films, or $2 \mathrm{mg} \mathrm{mL}^{-1}$ for HC LbL assembly. The resultant concentration of cleaved 2-pyridinethione was then measured using UV-Vis spectrophotometry against a calibration curve for 2-pyridinethione $\left(\lambda_{\max }=343 \mathrm{~nm}\right)$.

Fabrication of PMAsн Architectures. Planar PMAsH films were fabricated using a previously described method. ${ }^{9}$ For film fabrication on particle templates, a $\mathrm{SiO}_{2}(100$ $\mu \mathrm{L}, 50 \mathrm{~g} \mathrm{~L}^{-1}$ ) suspension was first washed in ethanol and Milli-Q, before being dispersed in $100 \mathrm{mM} \mathrm{NaOAc}$ buffer (pH 4). Three wash cycles (centrifugation followed by resuspension in $100 \mu \mathrm{L} \mathrm{NaOAc}$ ) were performed after the deposition of each polymer layer. For polymer deposition, $50 \mu \mathrm{L}$ of NaOAc buffer was first added, and the washed pellet resuspended via mixing. $\mathrm{PMAsH}_{(\mathrm{x})}$ and PVPON polymer solutions (50 $\mu \mathrm{L}, 2 \mathrm{mg} \mathrm{mL}^{-1}$ ) were then added, the solution mixed vigorously, and the polymer allowed to adsorb under mild agitation for $15 \mathrm{~min}$. This process was repeated until five $\mathrm{PMA}_{\mathrm{SH}(\mathrm{x})}$ layers were deposited. The resulting core-shell particles were then oxidized using CaT $(2.5 \mathrm{mM})$ in MES buffer ( $10 \mathrm{mM}, \mathrm{pH} 6)$ for $60 \mathrm{~s}$, resulting in the formation of disulfide cross-links. The particles were suspended in $20 \mu \mathrm{L} \mathrm{NaOAc}$, to which $80 \mu \mathrm{L}$ of $\mathrm{HF}(5 \mathrm{M}, \mathrm{pH}$ 1) was added. Caution! Hydrofluoric acid is very toxic. Extreme care should be taken when handling HF solution, and only small quantities should be prepared. The resulting capsules were washed four times via centrifugation $(4 \mathrm{~min}$, 
$4500 \mathrm{~g}$ ) and suspension in $100 \mu \mathrm{L}$ of $\mathrm{NaOAc}$ solution, followed by three centrifugation/wash cycles in $100 \mu \mathrm{L}$ of PBS solution (10 mM, pH 7.4). The resulting singlecomponent $\mathrm{PMA}_{\mathrm{SH}} \mathrm{HCs}$ were characterized using differential interference contrast (DIC) microscopy (Olympus IX71 microscope, Olympus $100 \times / 1.40$ Oil objective), and processed using ImageJ software and algorithms (NIH). ${ }^{34}$

AFM Cantilever Preparation. Both intermittent contact (IC) mode and CP cantilevers were cleaned to remove salt depositions and organics prior to use. The cantilevers were immersed in a $30 \mathrm{vol} \%$ isopropanol solution, followed by Milli-Q water, and then further cleaned using oxygen plasma for $180 \mathrm{~s}$ (Harrick Plasma, $0.1 \mathrm{~L} \mathrm{~min}^{-1} \mathrm{O}_{2}$ flow rate, 29.6 W, 300 mTorr). Glass substrates for cantilever spring constant calibration and capsule measurements were cleaned using the same method.

AFM Intermittent Contact Mode Imaging. For IC mode imaging of collapsed $\mathrm{HC}$ structures in air, standard tapping mode cantilevers with a spring constant of $40 \mathrm{~N} \mathrm{~m}^{-1}$ (Tap300-G, Budget Sensors, Bulgaria) were used. Images were recorded using a JPK Nanowizard II (JPK Instruments AG, Berlin, Germany). Images were post-treated using accompanying JPK image processing software and algorithms. A three-point $1^{\text {st }}$ degree polynomial fit was first subtracted, and then a polynomial fit subtracted from each scan line independently, excluding capsule regions.

AFM Force Spectroscopy. Force spectroscopy measurements were carried out in PBS (140 mM NaCl, pH 7.4) buffer using a JPK Nanowizard II (The University of Melbourne) for planar film and core-shell particles, and a JPK Nanowizard I (Bayreuth University) for HC measurements. Force measurements on planar PMAsH films, and CP cantilever calibration and preparation, have been described previously.9,35 For HC and core-shell particle measurements, PMAsн particles were immobilized onto a PEIcoated glass substrate. The glass substrate was cleaned with $30 \mathrm{vol} \%$ isopropanol and oxygen plasma prior to PEI adsorption. The sample was positioned above an Axiovert 200 microscope (Carl Zeiss MicroImaging, Jena, Germany) with Zeiss objectives $(20 \times$ and $63 \times$ oil). Measurements were conducted by aligning the probe over the centre of the visualized colloid, approaching the surface, and then engaging in a 'push-pull' cycle with the centre axis. This was achieved by lowering the cantilever until a predetermined force set-point was reached, and then raising the piezo back to its original position. For each measurement, the InvOLS of the PEI-coated glass substrate in working buffer was first determined to allow for accurate postprocessing of the force data. Approach speeds ranging from 0.3 to $1.5 \mu \mathrm{m} \mathrm{s}^{-1}$ were employed; however, the slopes of the HC force/deformation $(\mathrm{F} / \delta)$ curves were seen to be independent of approach speed between this range. Each push-pull cycle generated a force-displacement curve for treatment and analysis. Raw AFM data was processed using as received JPK software to subtract the zero-force baseline during cantilever approach, to set the origin to the initial contact point, and to extract data. The effect of cantilever bending during sample compression was removed using Equation 1 to give true sample deformation data,

$$
\delta=\text { Displacement }-\left(\mathrm{F} / \mathrm{k}_{\mathrm{c}}\right)
$$

where $k_{c}$ is the spring constant of the cantilever. For an incompressible material, the force increases with no observable change in deformation, while for softer samples the gradient reduces due to the compliance of the material. Due to possible viscoelastic effects and associated hysteresis, only the approach force curve was analyzed. The stiffness was evaluated to be the linear gradient of the force/deformation curve in the small-deformation regime, corresponding to deformations in the range of the wall thickness (approximately $40 \mathrm{~nm}$ ), as to negate the effects of capsule permeability. ${ }^{18}$ Reported stiffness values are an average of at least 20 measurements on separate capsules, with the error equating to one standard deviation of the data set. For the colloidal probe cantilevers used there was only a small variation in probe radius $(16.5 \pm 2.0 \mu \mathrm{m}$ for planar measurements, and $15.7 \pm 0.2 \mu \mathrm{m}$ for capsule measurements) and the effect of differences in contact area between samples was discounted. For the PMAsH(20) core-shell particles a probe of radius $16.2 \mu \mathrm{m}$ was used.

For investigating the force spectroscopy of films and capsules at variable $\mathrm{pH}$, the same experimental methodology was used as previously described. After measurement on the respective systems, ca. $50 \%$ of the $\mathrm{pH} 7.4$ buffer was carefully removed via micropipette aspiration to prevent drying, and an equal volume of $100 \mathrm{mM} \mathrm{NaOAc}$ buffer $(\mathrm{pH}$ 4.0) gently administered. This was repeated 10 times so that the resulting solution was at $\mathrm{pH} \sim 4.0$. The force measurements were then repeated at the new $\mathrm{pH}$ value.

Debye-Hückel Screening Length. The screening length $\left(\kappa^{-1}\right)$ was calculated to justify that negligible long range interactions occur between the colloidal silica probe and the sample. This can be evaluated using Equation 2,

$$
\kappa^{2}=4 \pi \lambda_{B} n
$$

where $n$ is the concentration of monovalent ions, and $\lambda_{B}$ is the Bjerrum length, which can be approximated as $0.7 \mathrm{~nm}$; the value for water at room temperature. Formally, $\lambda_{B}$ can be calculated using Equation 3,

$$
\lambda_{B}=\frac{e^{2}}{4 \pi \varepsilon_{0} \varepsilon_{\tau} k_{B} T}
$$

where $e$ is the elementary charge, $\varepsilon_{0}$ is the vacuum permittivity, $\varepsilon_{\tau}$ is the relative dielectric constant of the medium, $T$ the absolute temperature of the solution, and $k_{B}$ Boltzmann's constant. As we use a PBS buffer $\left(140 \mathrm{mM} \mathrm{NaCl}, \lambda_{B}\right.$ $\sim 0.7 \mathrm{~nm}$ ), we obtain a value for the Debye-Hückel screening length of approximately $0.9 \mathrm{~nm}$. As the range of indentations used for our measurements and analyses varied between 20 to $100 \mathrm{~nm}$, we can assume that nonzero range interactions in our buffered systems will be negligible. This result aligns with findings in literature for similar experiments. ${ }^{36}$

Applied Probe Axial Strain. As the cantilever probe applies a force to the material, it also exerts an intrinsic axial strain. It is important regarding linear elastic theory that an applied axial strain of 0.2 is not exceeded, otherwise material nonlinearity becomes an important factor. ${ }^{19}$ 
The axial strain $\left(\varepsilon_{z z}\right)$ can be evaluated using the active contact radius (a) during compression, where

$$
a=\left(\frac{3 F R_{e f f}\left(1-v^{2}\right)}{4 E_{Y}}\right)^{1 / 3}
$$

for the Poisson's ratio $(v)$, effective probe radius $\left(R_{e f f}\right)$, and the Young's modulus $\left(E_{Y}\right)$. This can then be substituted into the equation below which is developed from the differentiation of the axial displacement field $\left(u_{z}\right)$, where $\varepsilon_{z z}=\delta u_{z} / \delta z$, and

$$
\begin{aligned}
\varepsilon_{z z}(0,0) & =\frac{2 a}{\pi R_{e f f}(1-v)} \\
u_{z}(\mathbf{r}, z) & =\iint P_{z}\left(r_{s}\right) G(s) \cdot d A
\end{aligned}
$$

which can be evaluated according to the approach set out by Landau and Lifshitz. Here $P_{z}$ is the pressure distribution and $G(S)$ is the Green's function in $A$, the contact region. ${ }^{37}$ We can then model our colloidal-probe and capsule system to check the magnitude of the axial strain. When the applied axial strain is modeled for both planar films and capsule systems it is most sensitive for low Young's modulus and high Poisson's ratio systems. As it is important to only analyze data for applied forces that allow for an axial strain less than 0.2 , data collected for both planar and HC samples was only modeled to a maximum force of $1.0 \mathrm{nN}$. In the case of the low $E_{Y}$ PMAsh(5) samples, the maximum force studied regarding mechanical properties was $0.7 \mathrm{nN}$.

\section{RESULTS AND DISCUSSION}

PMA-PDA Characterization. The extent of crosslinking between neighboring $\mathrm{PMA}_{\mathrm{SH}}$ layers is governed by the number of thiol groups that are present in the polymer, which is equivalent to the number of PDA groups per PMA chain (Figure 1). PMA-PDA was synthesized using EDCactivated amide coupling chemistry. ${ }^{30}$ Target mole percentages of PMA-PDA of 5, 10, 15, and 20\% were prepared, and the resulting modified polymers denoted as $\mathrm{PMA}_{\mathrm{SH}}(5)$, PMAsh(10), PMAsh(15), and PMAsh(20), respectively (PMAsh(x)). The extent of PMA functionalization with PDA has been previously determined by comparing the integrals of the aromatic peaks with the peaks from the backbone of PMAPDA in corresponding ${ }^{1} \mathrm{H}-\mathrm{NMR}$ spectra. ${ }^{9}$ The amount of PDA present here was additionally quantified using UV-Vis spectrophotometry by measuring the concentration of 2pyridinethione $(343 \mathrm{~nm})$ that is released after reducing the PDA groups with DTT (pH 8) (Figure S1, Supporting Information). For all measured samples the UV-Vis characterization yields lower values by $30-40 \%$ of the PMA-PDA modification percentage, when compared to the values that were previously obtained using ${ }^{1} \mathrm{H}-\mathrm{NMR}$ spectroscopy (Figure S2, Supporting Information). It is likely that the PDA groups of PMA-PDA in aqueous solution are situated in the more hydrophobic regions of the polymer. This diminishes their reactivity and can lead to the mentioned quantification differences, whilst additionally affecting the actual degree of crosslinking occurring in PMAsH systems. Further, the contribution to the measured differences from small errors in the NMR integration cannot be excluded.
However, it is clear from both measurements that there is an increase in PDA substitution with target thiol modification.

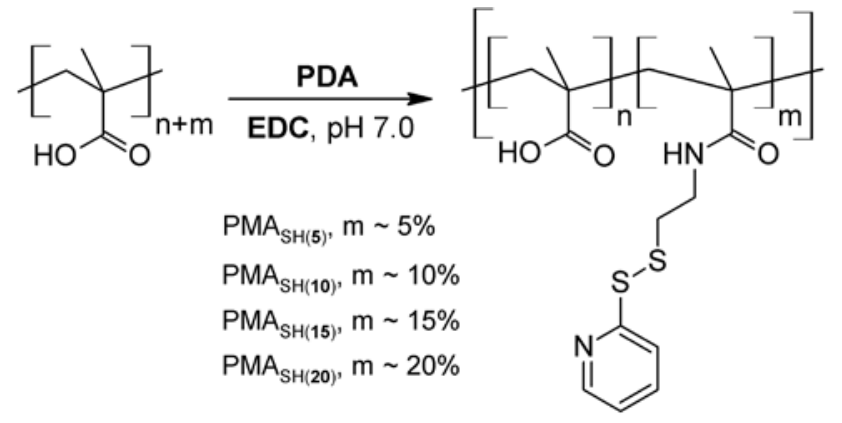

Figure 1. Preparation of PMA-PDA. The extent of pendant PDA modification was quantified using both ${ }^{1} \mathrm{H}-\mathrm{NMR}$ and UVVis spectrophotometry ( $m$ is the target thiol percentage).

Free-Standing PMAsн Capsules. We then investigated the formation of stable, free-standing, hydrogel capsules at physiological pH. PMAsh(x) polymers were used for LbL assembly of films on particle templates (Scheme 1). After the assembly of the planar multilayers, the thiol groups were cross-linked with CaT. Fabrication of free-standing $\mathrm{PMA}_{\text {sH }}$ capsules was achieved through removal of the silica core using HF, resulting in hollow shells for all modification percentages. Further washing into PBS solution at $\mathrm{pH}$ 7.4 resulted in removal of the PVPON by disrupting the Hbonds, as has been experimentally demonstrated in our previous work, ${ }^{28}$ to yield single-component PMA systems. Furthermore, it was observed that all HC systems fabricated were stable over a period of at least one week at physiological $\mathrm{pH}$. The resulting swollen capsules were characterized using DIC microscopy in PBS buffer. AFM imaging was used for air-dried capsules immobilized onto a PEI-coated substrate (Figure 2). DIC images in Figure 2 show that with increasing crosslinking degree the optical density of the HCs increases, along with a reduction in the degree of capsule swelling. AFM height image analysis using JPK-SPM data processing software showed that the shell thickness for the dried HCs increased with increasing thiol modification. If we assume that the shell volume is conserved for HC swelling, as proposed by Köhler et al., ${ }^{38}$ then the difference between theoretical shell thickness and measured dry thickness indicates a partial loss of material from the shell wall (Figure 2). Decreasing the number of cross-links leads to less stable hydrogel films, and non cross-linked material may be lost from the system; this aids capsule expansion at $\mathrm{pH}$ 7.4. A general trend was also observed from the AFM scans, where higher cross-linked HCs tended to display more folding features as a result of buckling than less cross-linked HCs. This indicates that higher cross-linked capsules are more rigid.

AFM force spectroscopy measurements were then performed to quantify the mechanical properties of the soft hydrogel materials using CP-AFM, and then to observe tuning of these properties through variation in crosslinking. In CP-AFM measurements, indentation of hydrogel systems is achieved through controlled piezo movement in 
the z-direction, with great precision (Figure 3A). As the probe is sensitive to force due to its large surface area, it is important to note that based on calculations for the DebyeHückel screening length for the colloidal silica probe in PBS, with $140 \mathrm{mM} \mathrm{NaCl}$, negligible long-range interactions occur between the probe and sample. For all force measurements there was negligible variation in the radius of the probe used for indentation, and axial strain was kept below 0.2 by analyzing forces $<1.0 \mathrm{nN}$ (Figure S3, Supporting Information), allowing us to treat deformations as being linearly elastic. A CP cantilever with a spring constant of $6.8 \mathrm{mN} \mathrm{m}^{-1}$ was used for PMAsh(5), while a cantilever with a constant of $66.4 \mathrm{mN} \mathrm{m}^{-1}$ was employed for the measurement of all other HCs. It was seen when collecting the AFM force data that approach and retract curves for the PMAsh(10), PMAsh(15), and PMAsh(20) capsule systems all showed considerable hysteresis.

It was previously observed that the deformation of melamine formaldehyde capsules generate hysteresis features, which generally occur for shell-based systems undergoing buckling transitions. ${ }^{22}$ The force response for PMAsH(5) HCs was primarily elastic, owing to the extremely soft and compliant nature of the system. Although the stiffer capsules demonstrated a shell-bending behavior, HCs were also multiple times to ca. $50 \%$ of their diameter, and it was seen that the force response was unchanged after at least 12 cycles, using a retracted delay time of $1.0 \mathrm{~s}$. This indicates that the HC compressions are elastic to at least 12 deformations, and that they retain their original shell conformation after each compression event.

An important requirement for performing force measurements on shells is that the deformation of the shell by the probe is performed uniaxially. ${ }^{39}$ This means that the capsule senses the force normal to its centre axis. As the diameter of the colloidal bead is approximately 30 times that of the HC, it was expected that the capsule would experience the

force 
similar to a parallel plate compression, thereby achieving uniaxial deformation over quite a large radial area. To test this, a $12 \times 12$ force map was performed over a $4 \times 4 \mu \mathrm{m}^{2}$ area where a single PMAsH(20) HC was immobilized. From Figure S4 (Supporting Information), the force curves collected at points A, B, C, and D give equivalent overall spectra with comparatively equal stiffness values $(14.0 \mathrm{mN} \mathrm{m}$ 1) over $40 \mathrm{~nm}$ of indentation, and are constant to $65 \mathrm{~nm}$ for spectra A, C, and D. It can therefore be assumed that, due to the relative sizes of the probe and capsule, uniaxial deformation can be achieved over a radius of approximately 2-3 $\mu \mathrm{m}$.

\section{Scheme 1. Fabrication of hydrogel architecturesa}
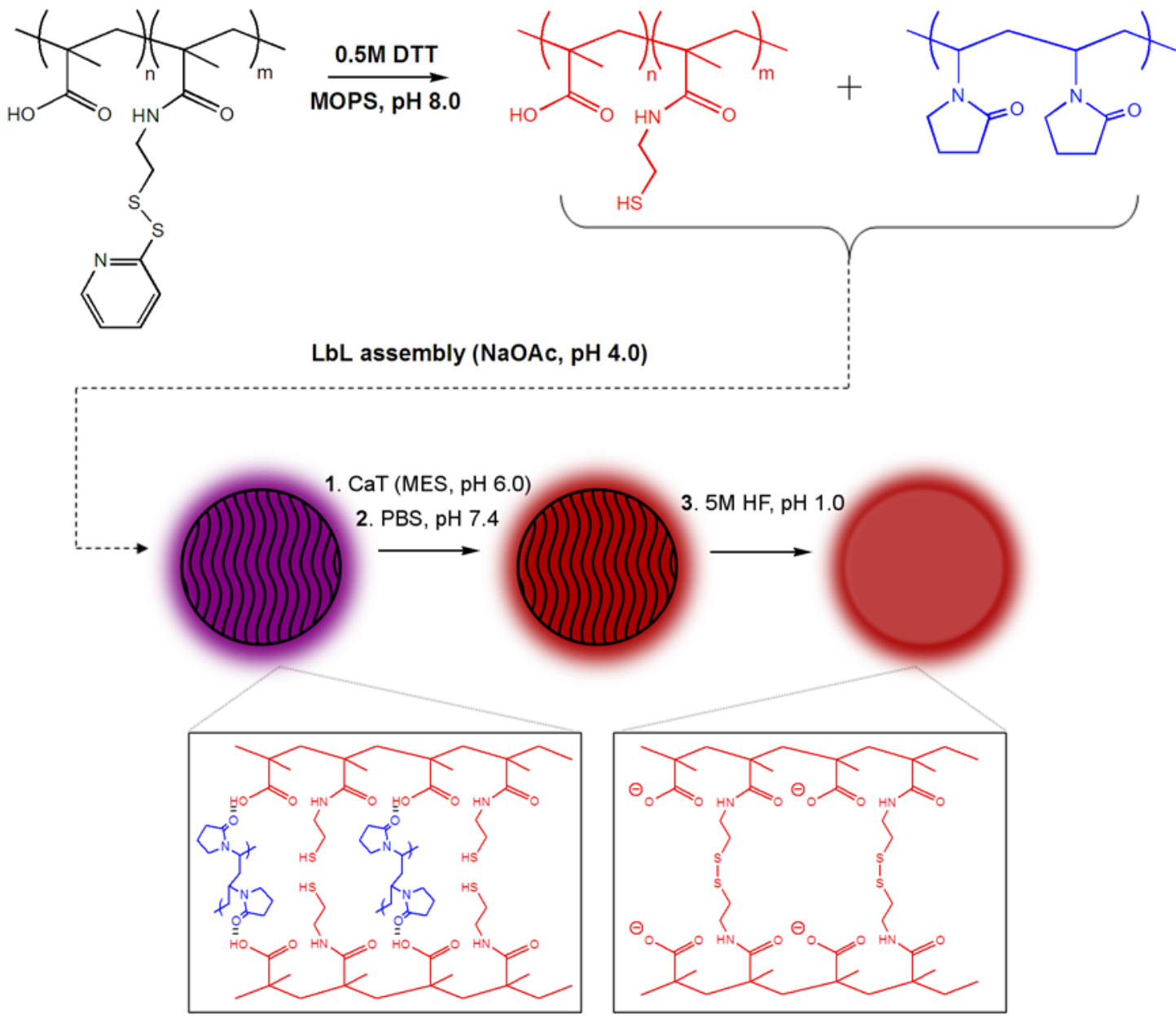

a Top: Reduction of PMA-PDA to PMAsh(x) (red). PMAsh(x) and PVPON (blue) are then assembled onto particulate silica templates at pH 4.0 (purple). $\mathrm{PMAsH}_{\mathrm{x}}$ is then oxidized to form stable disulfide cross-links, followed by removal of the PVPON with PBS at pH 7.4. Silica templates were then removed by dissolution using $5 \mathrm{M} \mathrm{HF}$ (magnifications show structure before crosslinking (left) and after resuspension in PBS at pH 7.4 (right)). 

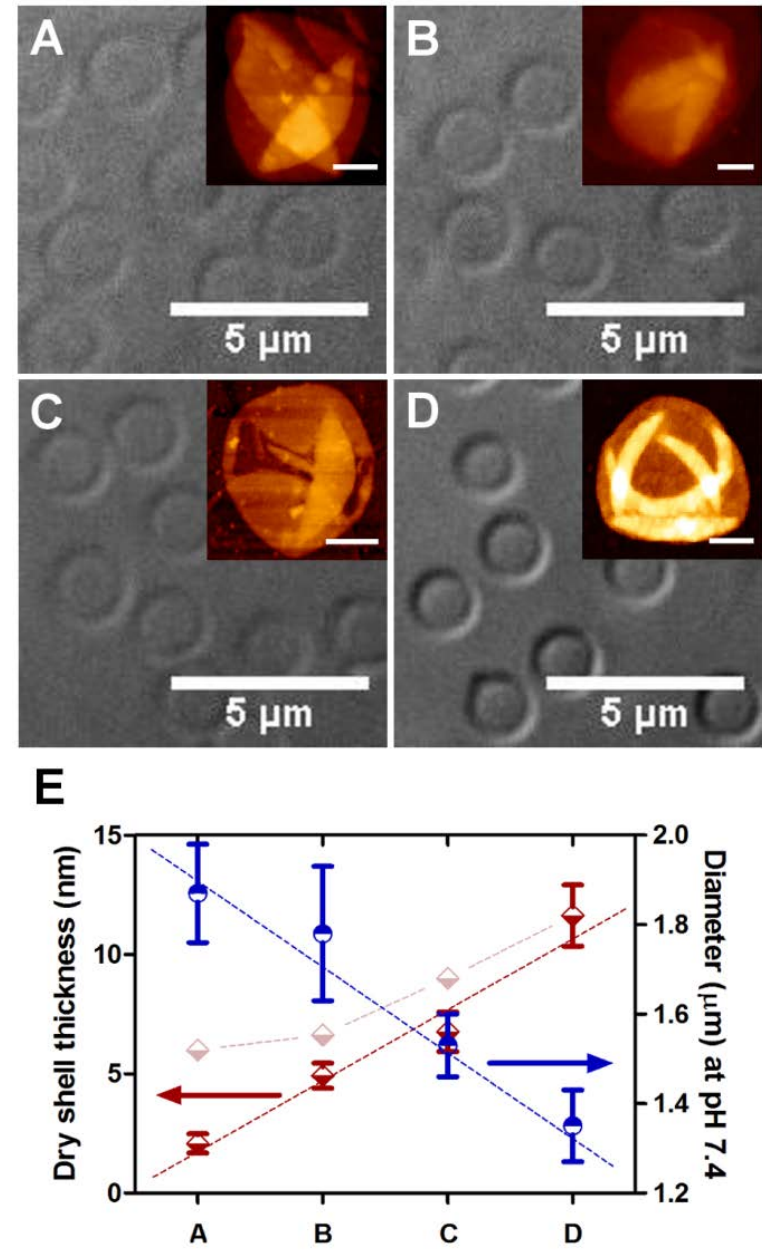

Figure 2. DIC microscopy images of HCs dispersed at pH 7.4. Insets show IC AFM scans of dried HCs immobilized onto a PEI-treated substrate (height range from 0 to $50 \mathrm{~nm}$, and scale bar $500 \mathrm{~nm}$, for all images). Images A, B, C and D represent PMAsh(5), PMAsh(10), PMAsh(15), and PMAsh(20) HCs, respectively. HC diameter at $\mathrm{pH} 7.4$ from DIC microscopy analysis (blue circles), air-dried HC shell thickness (red diamonds) from AFM imaging analysis, and theoretical air-dried shell thickness assuming conservation of shell volume (transparent red diamonds) (E). Error bars represent one standard deviation, and dotted lines are used to guide the eye only.

Analyzing the small-deformation gradient for the HCs (Figure 3B) allowed for determination of the shell stiffness. While the HCs could be tuned between 0.9 and $16.9 \mathrm{mN} \mathrm{m}^{-}$ 1 for 5 to $20 \mathrm{~mol} \%$ PDA modification, we previously reported that planar hydrogel films could be tuned between 0.7 and $5.7 \mathrm{mN} \mathrm{m}^{-1}$ over the same range. ${ }^{9}$ A quadratic increase was observed for the HC structures, whereas the planar film stiffness increased linearly. The basis of this relationship for the $\mathrm{HC}$ results can be observed in the Reissner model for thin spherical shells, where the capsule stiffness is proportional to the square of the shell thickness for an isotropic elastic material (Equation 7). ${ }^{40}$ This relationship is evident in the data contained in both Figures 2 and 3 , where a quadratic relationship must exist between HC stiffness and dry shell thickness, similar to trends ob- served in literature. ${ }^{39}$ The hydrated shell thickness of low cross-linked HCs is difficult to quantify accurately. As shown in previous work, ${ }^{9}$ low cross-linked films that experience contraction during dehydration are fragile and lose material. Collapsing a low cross-linked HC in air and then washing and rehydrating in buffer would result in removal of a portion of the wall material, leading to inaccuracies in determining the wall thickness. In addition, once a capsule collapses there is increased electrostatic interaction between the PMAsн and surface-bound PEI.

$$
E_{Y, \text { Reissner }}=\frac{F R_{\text {eff }} \sqrt{3\left(1-v^{2}\right)}}{4 \delta h^{2}}
$$

For estimation of the Young's modulus, we assumed that all HCs had an equal hydrated shell volume, and that the shell thickness would reduce as the capsules swell with decreasing crosslinking degree. This relationship was demonstrated by Köhler et al. for the thermal shrinking of polyelectrolyte capsules, where it was shown that conservation of the shell volume occurred. ${ }^{38}$ We also employed the Reissner relationship for thin shells (Equation 7), which has been used previously for modeling the $E_{Y}$ of polyelectrolyte capsule shells. ${ }^{41}$ The applicability of thin shell theory, assumed in the Reissner model, is often limited to a shell thickness to radius ratio of less than $1 / 20.42$ Collapsed PMAsH(20) HCs have a hydrated shell thickness in PBS buffer of roughly $40 \mathrm{~nm}$ (data not shown), with a diameter of $1.35 \mu \mathrm{m}$ in free suspension. This is equivalent to $h / R \sim 0.06$, greater than the $1 / 20$ limit discussed. Indeed, Mercadé-Prieto et al. utilized finite element modeling to demonstrate that Equation 7 only applies to thin shells dependent on bending deformations, and that thicker shells require corrections due to considerable Green and Hencky strains. ${ }^{43}$

For $E_{Y}$ evaluation using the Reissner model, $R_{\text {eff }}$ was calculated as a function of both $\mathrm{CP}$ and $\mathrm{HC}$ radial components. As it was not possible to accurately measure the hydrated shell thickness, $h$ was estimated based on shell volume conservation. More weakly cross-linked HCs expanded at $\mathrm{pH} 7.4$, thereby decreasing the shell thickness, assuming an equal initial mass of polymer adsorbed to the surface for all systems. Using these assumptions, the thickness for the $\mathrm{PMA}_{\mathrm{SH}(20)}$ system was estimated, and the shell thickness for the other HC systems based on this. With these values, the Young's modulus of the HCs was found to increase from 0.70 to $2.56 \mathrm{MPa}$. In literature, comparable free-standing systems were shown to exhibit elastic moduli typically in the low GPa range. ${ }^{21}$ For instance, Glynos et al. found that polylactide microspheres with a cross-linked albumin outer skin had a shell stiffness of $3.8 \mathrm{~N} \mathrm{~m}^{-1}$, while the $E_{Y}$ was evaluated to be $2.5 \mathrm{GPa}$ using the Reissner relationship. ${ }^{15}$ In contrast, our HCs prove to be remarkably soft, and of a similar magnitude to reported collapsed cross-linked PMA capsules evaluated using a Hertz model. ${ }^{25}$

Influence of $\mathbf{p H}$ on Film Mechanics. Next, we investigated the potential effect of net shell 'pressurization' on the observed enhanced force resistance of the HCs. As this effect would be due to PMAsH charge repulsion in the shell, we studied the swelling behavior and stiffness characteris- 
tics of PMAsH(15) capsules at both $\mathrm{pH} 7.4$ and 4.0. As shown in Figure 4, the capsule stiffness increases considerably when the $\mathrm{pH}$ is dropped to 4.0. It is postulated that if the shell demonstrated a pressurization effect, decreasing the $\mathrm{pH}$ and removing the

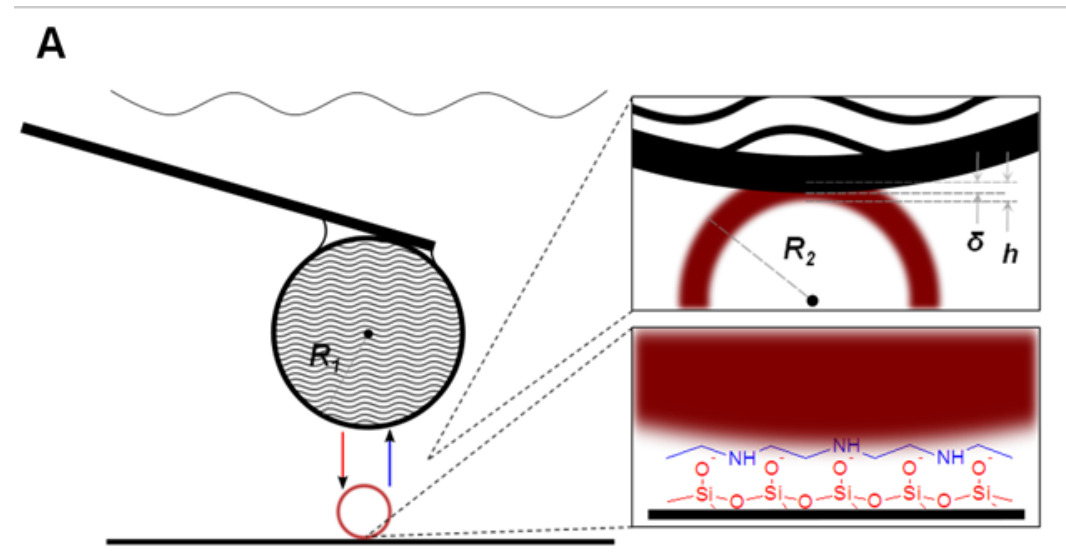

B

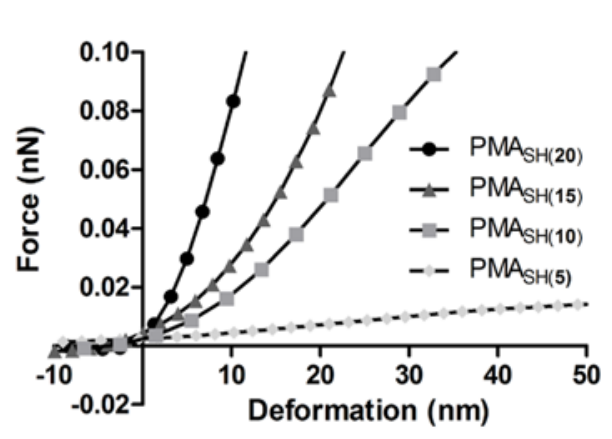

Figure 3. Schematic representation of the CP-AFM technique (A) and representative small-deformation $F / \delta$ curves for immobilized HCs (B). An attached glass microsphere (radius $R_{1}$ ) is positioned above an immobilized HC in PBS buffer, from where approach (red arrow) and retract (blue arrow) force curves are generated (A). Top inset depicts deformation $(\delta$ ) of the HC (shell thickness $h$, radius $R_{2}$ ) by the CP, while the bottom inset illustrates surface immobilization of the HC using PEI (blue).

A

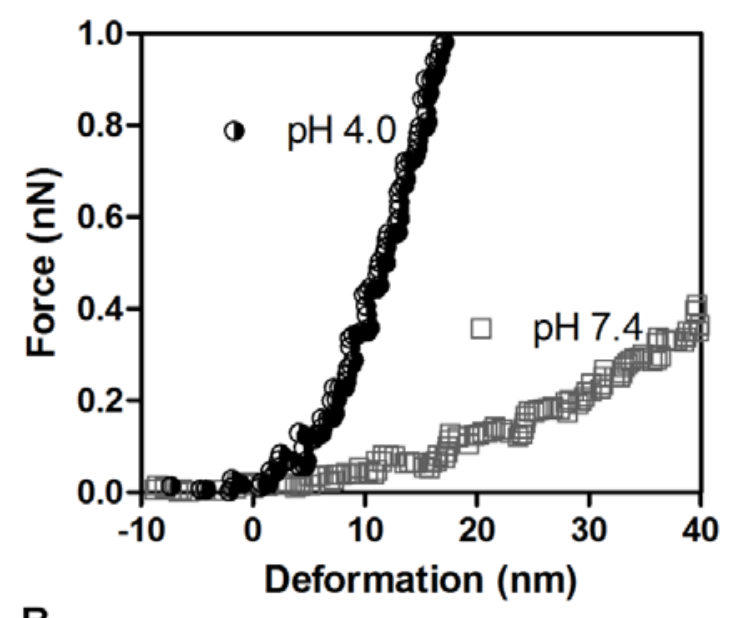

B

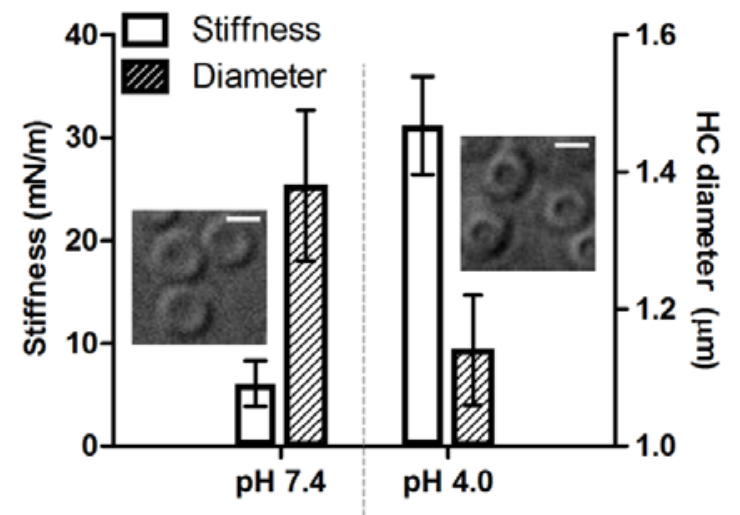

Figure 4. Influence of $\mathrm{pH}$ on $\mathrm{PMAsH}(15) \mathrm{HC}$ stiffness. Representative force curves (A), DIC microscope images, and stiffness and diameter results (B) for PMAsH(15) capsules at both pH 7.4 and pH 4.0. Error bars in B correspond to one standard deviation, while scale bars for the DIC images equal $1 \mu \mathrm{m}$.

charge repulsion would soften the capsules. As an increase in stiffness is observed with reduced HC swelling, the effect of shell pressurization on force resistance is negligible when compared to other factors.

We then correlated this finding to the $\mathrm{pH}$ behavior of $\mathrm{PMA}_{\mathrm{sH}}$ films fabricated on solid-supported planar structures. PMAsh(20) was used for this experiment as it has previously demonstrated a high degree of stability, so any differences in mechanical properties of the film at different $\mathrm{pH}$ values is a function of film behavior rather than possible film disruption during buffer change. Force experiments were performed as per the other PMAsH film measurements; ${ }^{9}$ however, after the initial measurement, the $\mathrm{pH}$ 7.4 buffer was replaced with buffer at $\mathrm{pH} 4.0$, and the force map repeated. It was observed that the film thickness decreased to ca. $35 \mathrm{~nm}$, and that the stiffness and $E_{Y}$ both increased roughly 20 -fold (Figure 5). We had previously observed a general film thickness of $130 \pm 50 \mathrm{~nm}$ for all $\mathrm{PMA}_{\mathrm{sH}(\mathrm{x})}$ films, ${ }^{9}$ where the film thickness was evaluated as the deformation distance of the probe, from initial contact to substrate incompressibility, based on a literature technique. ${ }^{44}$ Sukhishvili and coworkers previously found that EDC cross-linked PMA films also contracted greatly when the $\mathrm{pH}$ was reduced. ${ }^{24}$ This can also be related to results published by Elsner et al. for PMA capsules, where it was found that as the solution $\mathrm{pH}$ decreased below the $\mathrm{pKa}$ of the polymer system, the stiffness increased dramatically due to the formation of hydrogen bonding interactions. ${ }^{23}$ 
These reports are consistent with our observations that a reduction of the solution $\mathrm{pH}$ below the $\mathrm{pKa}$ of $\mathrm{PMA}_{\mathrm{sH}}$ increases the film density due to the carboxyl group protonation on the polymer backbone.

PMAsh Films Anchored on Particulate Supports. As an intermediate step to the production of free-standing hollow capsules, we examined whether mechanical differences between HCs and planar films may occur due to film formation on particle substrates. The LbL technique was used to adsorb alternating layers of PVPON and PMAsh(20) onto particulate silica templates $(d=1.11 \mu \mathrm{m})$ in NaOAc buffer. The hydrogel core-shell particles were cross-linked, dispersed into PBS buffer and then immobilized onto a PEI-coated glass substrate. Measurements were performed on immobilized colloids visualized optically; the probe positioned above the center axis of the colloid to achieve uniaxial deformation, and an approach/retract cycle initiated to measure small material deformations of the film as a function of the applied force. Immobilization is an important aspect of the measurement, as insufficient immobilization can lead to lateral movement during force application, and result in poor or misleading spectra. The material stiffness in the small deformation regime was determined from the treated spectra and found to be $2.1 \pm 0.4 \mathrm{mN} \mathrm{m}^{-1}$, approximately half that of PMAsh(20) planar films on mica, as shown in Figure 6. This is an interesting observation, as we would expect core-shell particles to be deformed at two contact points when under an applied force: at both the substrate and colloidal probe surfaces. This would lead to a material stiffness half that of the planar hydrogel films, as an incompressible spherical substrate allows for no bending resistance. A further explanation for this was given by Fytas and coworkers when they observed that poly(methyl methacrylate) (PMMA) capsules had different mechanical properties to $\mathrm{SiO}_{2}$-PMMA core-shell particles. ${ }^{45}$ Using light scattering techniques, it was postulated that these differences arose due to differences in the system architecture.

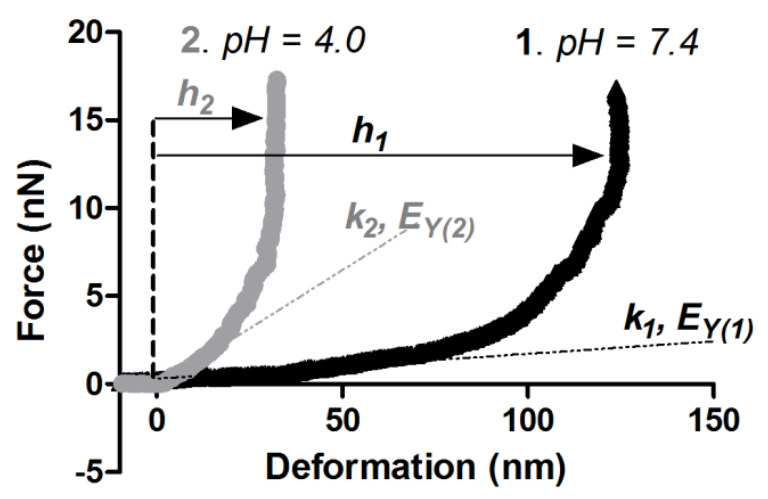

Figure 5. The effect of in situ pH variation on PMAsh(20) film thickness $(h)$ and mechanical properties $\left(k, E_{Y}\right)$ measured using CP-AFM.
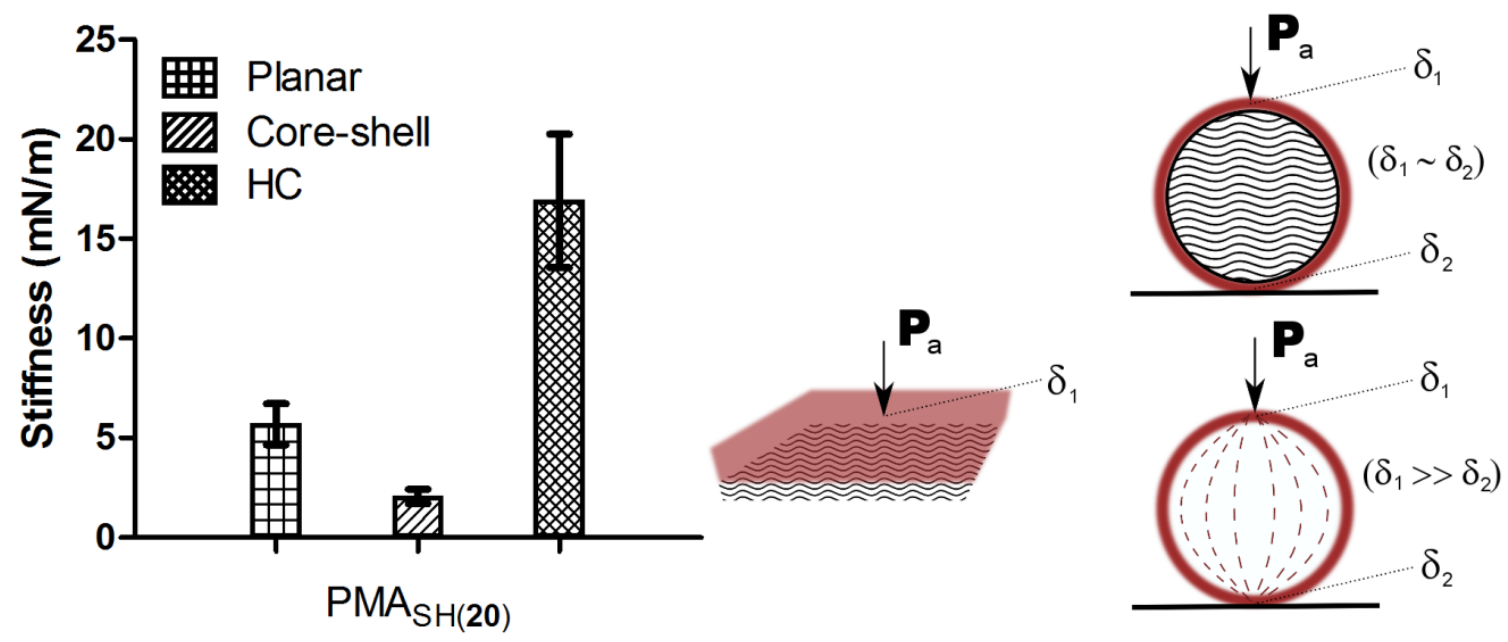

Figure 6. Stiffness values for PMAsh(20) hydrogels confined in planar film, core-shell, and HC architectures. An applied force $\left(P_{a}\right)$ affects a deformation $(\delta)$ of the film surface. Error bars represent one standard deviation (planar film results reproduced from ref. 9). 
Transition from Planar Films to Hollow Capsules. We previously reported on the mechanical properties of PMAsH planar films on atomically flat mica substrates. ${ }^{9}$ It was found that the stiffness of the hydrogel films could be effectively tuned through adjustment of the degree of disulfidebased crosslinking, and that all films were mechanically homogeneous over a $50 \mu^{2}$ area. From 5 to $20 \mathrm{~mol} \%$ PDA modification, we reported that the stiffness of the films was found to increase from 0.7 to $5.7 \mathrm{mN} \mathrm{m}^{-1}$, while the $E_{Y}$ increased from 0.4 to $2.1 \mathrm{kPa}$ using a Hertz model, or 1.0 to $2.3 \mathrm{kPa}$ evaluated using the Johnson-Kendall-Roberts (JKR) model for adhesive contact. ${ }^{9}$ All mechanical results were observed to increase linearly with increasing PDA modification. Comparable materials in literature characterized using CP-AFM measurements demonstrate that planar $\mathrm{PMA}_{\mathrm{sH}}$ films are extremely soft. Using Hertz modeling, soft gelatin films were found to have an $E_{Y}$ of $20 \mathrm{kPa}$ to $1 \mathrm{MPa},{ }^{10}$ while both the Picart and Singer groups found that using EDC as a cross-linker, thin hydrogel films could have their modulus tuned between $3 \mathrm{kPa}$ to $1 \mathrm{MPa} .^{20,46}$

In this article we have shown that the elastic modulus determined for HCs based on Reissner's theory is three orders of magnitude greater than those for planar films of the same material. It would generally be assumed that these ranges would be similar, as the material is constant. A possible explanation for these differences was provided by Glynos and coworkers, based on their observation that thick shelled polymer microspheres had an $E_{Y}$ approaching that of the bulk material, whereas thinner shelled spheres had a dramatically increased $E_{Y}{ }^{47}$ They postulated that morphological and molecular restructuring may occur due to confinement in thinner shells, and that as the dimensions approach the nanoscale, surface effects such as surface tension and gradient elasticity can have a profound influence on material properties. ${ }^{47}$ In addition, the Reissner model is not directly applicable to the PMA $A_{S H}$ HC systems studied. The shell thickness and small diameter of the HCs dictates that they are at, or above, the limit of thin shell theory required for use of the Reissner model. Above this limit estimates of $E_{Y}$ are reliant on significant corrections, and accuracy is dependent on accurate shell thickness information.43 Other assumptions in these models were met, however, as uniaxial deformation and low applied axial strain was achieved during measurement. In addition, this result highlights the requirement of contact mechanics models for soft, viscoelastic, nanostructured hydrogel shells.

The increased HC stiffness observed when compared to planar films fabricated using the same PMAsh(x), as is seen in Figure 7, is of inherent interest. During HC compression, system deformation may occur at the two contact points on the shell surface; however, this will occur predominantly at the CP contact point due to finite energy dissipation through the shell structure via bending resistances. The CP cantilever measures resistance to applied force through both the film and shell bending resistances, if we assume that the HC is not instantaneously permeable to the encapsulated solvent. This would increase the stiffness felt by the cantilever when compared to the single contact point indentation for films, which allow for increased energy dissipation, as they can be viewed as essentially an infinite plane. Geometrically, spheres have the smallest surface area for a given volume, and have a maximized moment of inertia increase around the shell bending axis. This gives a spherical shell structure an increased apparent stiffness when compared to an immobilized membrane of the same material and shell thickness. Further, to our knowledge, planar materials with a system stiffness $<5 \mathrm{mN} \mathrm{m}^{-1}$ have not been characterized in the literature, and only one example of similarly soft HCs has been shown, ${ }^{23}$ demonstrating the applicability of CP-AFM for this task.

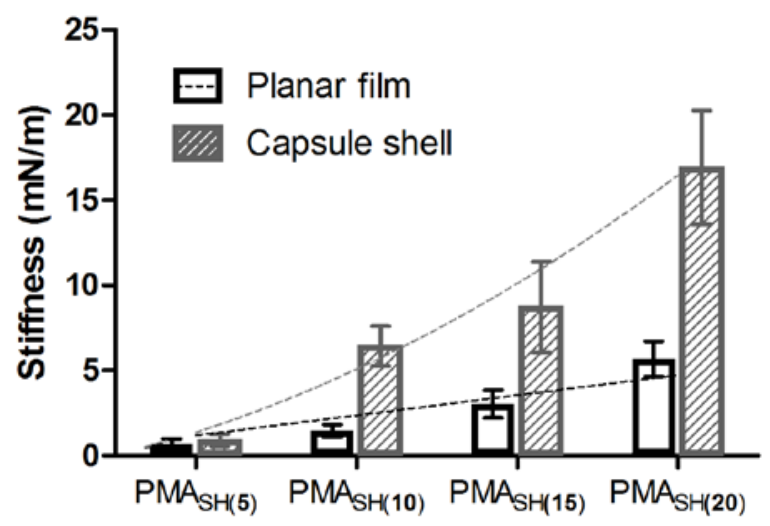

Figure 7. Stiffness comparison results for planar hydrogel films on mica substrates (planar film results reproduced from ref. 9), and PEI immobilized HCs. Error bars represent one standard deviation. Planar film data is fit with a linear regression, while capsule shell stiffness data is fit with a 2nd order polynomial. Error bars represent one standard deviation.

As shown in Figure 7, the planar film stiffness increases linearly with crosslinking degree, as the network becomes increasingly dense and robust to external force. HC stiffness increases at a greater rate with crosslinking, as both the shell becomes more robust and the capsules resist expansion. The increase in stiffness observed for the HCs is a combination of both enhanced material stiffness (shown through increases in film stiffness), and consolidated shell structure (verified based on the observed increases in shell thickness). Previous analyses of PSS/PAH multilayers in planar and capsule configurations demonstrated that capsules tend to have a greater $E_{Y}\left(1.5\right.$ to $2.0 \mathrm{GPa},{ }^{48}$ and 1.3 to $\left.1.9 \mathrm{GPa}^{14}\right)$ when compared to planar films $(0.30$ to 0.59 $\mathrm{GPa}$ ) measured using an elastic buckling technique. ${ }^{49}$ Bathe and coworkers were able to show through finite element analysis that the ratio of membrane to bending energies stored within a shell system was highly sensitive to the shell thickness. ${ }^{50}$ These results support the argument that the shell structure of PMAsH HCs aids in improving the mechanical stiffness of the system. This finding can be reasoned when considering the bending energies of thin shells and spherical vessels under an applied pressure. Based on the Kirchhoff-Love plate theory for elastic shells, the ener- 
gy change derived from a differential shell geometry is made up of both membrane and bending strains. ${ }^{51}$ Due to increased stiffness values for the HCs, we were able to observe these shell resistances for micron-sized soft hydrogel capsules when compared to planar films of the same material.

\section{CONCLUSIONS}

The mechanical properties of PMAsh(x) free-standing hollow capsules with varying degrees of crosslinking were investigated. The stability of these constructs was analyzed using optical and AFM techniques, and it was found that introducing a higher degree of disulfide crosslinking improved both network density and stability. The material compliance was quantified using CP-AFM, and it was found that a hollow capsule architecture offers a greater force resistance when compared to substrate-supported (planar and particulate) films of the same material. It was postulated that this was due to the transference of the applied force to bending energies within the capsule structure, geometric effects, and shell densification. The mechanical properties of the PMASH systems were quantified using the highly sensitive CP-AFM technique, and results indicated that they were extremely soft and tunable via network density.

We envisage that these results will be useful for researchers involved in the study and characterization of soft and tunable hydrogel shells, in particular for studies where particle material parameters are assumed based on planar film studies. Future work will examine the biological interaction of these materials, and the effect that compliance has upon effects such as cellular association and uptake, in vivo clearance rates, cellular adhesion strength, and modulation of biomolecule/conjugate interactions.

\section{ASSOCIATED CONTENT}

Supporting Information. UV-Vis spectra for PMA-PDA characterization, applied probe axial strain modeling, and uniaxial deformation AFM force map and spectra for HCs. This material is available free of charge via the Internet at http://pubs.acs.org.

\section{AUTHOR INFORMATION}

\section{Corresponding Author}

* Prof. F. Caruso: E-mail fcaruso@unimelb.edu.au, Tel +61-38344-3461, Fax +61-3-8344-4153. Prof. A. Fery: E-mail andreas.fery@uni-bayreuth.de, Tel +49-0-921/55-2753, Fax +49-0-921/55-2059.

\section{Author Contributions}

The manuscript was written through contributions of all authors. All authors have given approval to the final version of the manuscript.

\section{Notes}

The authors declare no competing financial interest.

\section{ACKNOWLEDGMENT}

This work was supported by the Australian Research Council under the Australian Laureate Fellowship (F.C., 120100030), Discovery Project schemes (F.C., 130101846), and the European Union-funded Nano-sirna Project (A.F.; F.C.).

\section{ABBREVIATIONS}

AFM, atomic force microscopy; CaT, $N$-chloro- $p$ toluenesulfonamide sodium salt; CP, colloidal-probe; DIC, differential interference contrast; DTT, dithiothreitol; EDC, 1ethyl-3-(3-dimethylaminopropyl) carbodiimide; HC, hydrogel capsule; HF, hydrofluoric acid; IC, intermittent contact; InvOLS, inverse optical lever sensitivity; JKR, Johnson-KendallRoberts; LbL, layer-by-layer; MES, 2-( $N$-morpholino) ethanesulfonic acid; MOPS, 3-( $N$-morpholino) propanesulfonic acid; MWCO, molecular weight cut-off; NaOAc. sodium acetate; NMR, nuclear magnetic resonance; PBS, phosphate buffered saline; PDA, 2-(2-pyridinyldithio) ethaneamine; PEI, polyethyleneimine; PMA, poly(methacrylic acid); PMA-Na, poly(methacrylic acid, sodium salt); $\mathrm{PMAsH}_{\mathrm{s}}$, thiol modified poly(methacrylic acid); PMMA, poly(methyl methacrylate); ppm, parts per million; PVPON, poly( $N$-vinyl pyrrolidone); QCM-D, quartz crystal microgravimetry; UV-Vis, UltravioletVisible.

\section{REFERENCES}

(1) Hoare, T. R.; Kohane, D. S. Hydrogels in Drug Delivery: Progress and Challenges. Polymer 2008, 49, 1993-2007.

(2) Becker, A. L.; Johnston, A. P. R.; Caruso, F. Layer-By-LayerAssembled Capsules and Films for Therapeutic Delivery. Small 2010, 6, 1836-1852.

(3) Nguyen, K. T.; Shukla, K. P.; Moctezuma, M.; Braden, A. R. C.; Zhou, J.; Hu, Z.; Tang, L. Studies of the Cellular Uptake of Hydrogel Nanospheres and Microspheres by Phagocytes, Vascular Endothelial Cells, and Smooth Muscle Cells. J. Biomed. Mater. Res. Part A 2009, 88A, 1020-1030.

(4) Stuart, M. A. C.; Huck, W. T.; Genzer, J.; Müller, M.; Ober, C.; Stamm, M.; Sukhorukov, G. B.; Szleifer, I.; Tsukruk, V. V.; Urban, M. Emerging Applications of Stimuli-Responsive Polymer Materials. Nat. Mater. 2010, 9, 101-113.

(5) Best, J. P.; Yan, Y.; Caruso, F. The Role of Particle Geometry and Mechanics in the Biological Domain. Adv. Healthcare Mater. 2012, 1, 35-47.

(6) Yan, Y.; Such, G. K.; Johnston, A. P. R.; Best, J. P.; Caruso, F. Engineering Particles for Therapeutic Delivery: Prospects and Challenges. ACS Nano 2012, 6, 3663-3669.

(7) Banquy, X.; Suarez, F.; Argaw, A.; Rabanel, J.-M.; Grutter, P.; Bouchard, J.-F.; Hildgen, P.; Giasson, S. Effect of Mechanical Properties of Hydrogel Nanoparticles on Macrophage Cell Uptake. Soft Matter 2009, 5, 3984-3991.

(8) Georges, P. C.; Janmey, P. A. Cell Type-Specific Response to Growth on Soft Materials. J. Appl. Physiol. 2005, 98, 1547-1553.

(9) Best, J. P.; Javed, S.; Richardson, J. J.; Cho, K. L.; Kamphuis, M. M. J.; Caruso, F. Stiffness-Mediated Adhesion of Cervical Cancer Cells to Soft Hydrogel Films. Soft Matter 2013, 9, 4580-4584.

(10) Domke, J.; Radmacher, M. Measuring the Elastic Properties of Thin Polymer Films with the Atomic Force Microscope. Langmuir 1998, 14, 3320-3325.

(11) Kovalev, A.; Shulha, H.; Lemieux, M.; Myshkin, N.; Tsukruk, V. Nanomechanical Probing of Layered Nanoscale Polymer Films with Atomic Force Microscopy. J. Mater. Res. 2004, 19, 716-728.

(12) Markutsya, S.; Jiang, C.; Pikus, Y.; Tsukruk, V. V. Freely Suspended Layer-By-Layer Nanomembranes: Testing Micromechanical Properties. Adv. Funct. Mater. 2005, 15, 771780. 
(13) Schmidt, S.; Zeiser, M.; Hellweg, T.; Duschl, C.; Fery, A.; Möhwald, H. Adhesion and Mechanical Properties of PNIPAM Microgel Films and Their Potential Use as Switchable Cell Culture Substrates. Adv. Funct. Mater. 2010, 20, 3235-3243.

(14) Dubreuil, F.; Elsner, N.; Fery, A. Elastic Properties of Polyelectrolyte Capsules Studied by Atomic-Force Microscopy and RICM. Eur. Phys. J. E 2003, 12, 215-221.

(15) Glynos, E.; Sboros, V.; Koutsos, V. Polymeric Thin Shells: Measurement of Elastic Properties at the Nanometer Scale Using Atomic Force Microscopy. Mater. Sci. Eng. B Adv. Funct. Solid-State Mater. 2009, 165, 231-234.

(16) Ye, C.; Drachuk, I.; Calabrese, R. Dai, H.; Kaplan, D. L.; Tsukruk, V. V. Permeability and Micromechanical Properties of Silk Ionomer Microcapsules. Langmuir 2012, 28, 12235-12244.

(17) Ducker, W. A.; Senden, T. J.; Pashley, R. M. Direct Measurement of Colloidal Forces Using an Atomic Force Microscope. Nature 1991, 353, 239-241.

(18) Fery, A.; Dubreuil, F.; Möhwald, H. Mechanics of Artificial Microcapsules. New J. Phys. 2004, 6, 18-30.

(19) Dimitriadis, E. K.; Horkay, F.; Maresca, J.; Kachar, B.; Chadwick, R. S. Determination of Elastic Moduli of Thin Layers of Soft Material Using the Atomic Force Microscope. Biophys. J. 2002, 82, 2798-2810.

(20) Francius, G.; Hemmerle, J.; Ohayon, J.; Schaaf, P.; Voegel, J. C.; Picart, C.; Senger, B. Effect of Crosslinking on the Elasticity of Polyelectrolyte Multilayer Films Measured by Colloidal Probe AFM. Microsc. Res. Techniq. 2006, 69, 84-92.

(21) Pretzl, M.; Neubauer, M.; Tekaat, M.; Kunert, C.; Kuttner, C.; Leon, G. r.; Berthier, D.; Erni, P.; Ouali, L.; Fery, A. Formation and Mechanical Characterization of Aminoplast Core/Shell Microcapsules. ACS Appl. Mater. Interfaces 2012, 4, 2940-2948.

(22) Lulevich, V. V.; Andrienko, D.; Vinogradova, O. I. Elasticity of Polyelectrolyte Multilayer Microcapsules. J. Chem. Phys. 2004, $120,3822-3826$

(23) Elsner, N.; Kozlovskaya, V.; Sukhishvili, S. A.; Fery, A. pHTriggered Softening of Crosslinked Hydrogen-Bonded Capsules. Soft Matter 2006, 2, 966-972.

(24) Kozlovskaya, V.; Kharlampieva, E.; Mansfield, M. L.; Sukhishvili, S. A. Poly(methacrylic acid) Hydrogel Films and Capsules: Response to $\mathrm{pH}$ and Ionic Strength, and Encapsulation of Macromolecules. Chem. Mater. 2006, 18, 328-336.

(25) Drachuk, I.; Shchepelina, O.; Lisunova, M.; Harbaugh, S.; Kelley-Loughnane, N.; Stone, M.; Tsukruk, V. V. pH-Responsive Layer-by-Layer Nanoshells for Direct Regulation of Cell Activity. ACS Nano 2012, 6, 4266-4278.

(26) Becker, A. L.; Zelikin, A. N.; Johnston, A. P. R.; Caruso, F. Tuning the Formation and Degradation of Layer-by-Layer Assembled Polymer Hydrogel Microcapsules. Langmuir 2009, 25, 14079-14085.

(27) Chong, S. F.; Lee, J. H.; Zelikin, A. N.; Caruso, F. Tuning the Permeability of Polymer Hydrogel Capsules: An Investigation of Cross-Linking Density, Membrane Thickness, and Cross-Linkers. Langmuir 2011, 27, 1724-1730.

(28) Zelikin, A. N.; Li, Q.; Caruso, F. Disulfide-Stabilized Poly(methacrylic acid) Capsules: Formation, Cross-Linking, and Degradation Behavior. Chem. Mater. 2008, 20, 2655-2661.

(29) Saito, G.; Swanson, J. A.; Lee, K.-D. Drug Delivery Strategy Utilizing Conjugation via Reversible Disulfide Linkages: Role and Site of Cellular Reducing Activities. Adv. Drug Delivery Rev. 2003, $55,199-215$.

(30) Zelikin, A. N.; Quinn, J. F.; Caruso, F. Disulfide Cross-Linked Polymer Capsules: En Route to Biodeconstructible Systems. Biomacromolecules 2005, 7, 27-30.

(31) Shimoni, O.; Postma, A.; Yan, Y.; Scott, A. M.; Heath, J. K.; Nice, E. C.; Zelikin, A. N.; Caruso, F. Macromolecule Functionalization of Disulfide-Bonded Polymer Hydrogel Capsules and Cancer Cell Targeting. ACS Nano 2012, 6, 1463-1472.
(32) Zelikin, A. N.; Li, Q.; Caruso, F. Degradable Polyelectrolyte Capsules Filled with Oligonucleotide Sequences. Angew. Chem. Int. Ed. 2006, 45, 7743-7745.

(33) Zelikin, A. N.; Becker, A. L.; Johnston, A. P. R.; Wark, K. L.; Turatti, F.; Caruso, F. A General Approach for DNA Encapsulation in Degradable Polymer Microcapsules. ACS Nano 2007, 1, 63-69.

(34) Abramoff, M. D.; Magelhaes, P. J.; Ram, S. J. Image Processing with ImageJ. Biophotonics Int. 2004, 11, 36-42.

(35) Attard, P. Measurement and Interpretation of Elastic and Viscoelastic Properties with the Atomic Force Microscope. J. Phys. Condens. Matter 2007, 19, 473201-473233.

(36) Francius, G.; Hemmerlé, J.; Ohayon, J.; Schaaf, P.; Voegel, J.C.; Picart, C.; Senger, B. Effect of Crosslinking on the Elasticity of Polyelectrolyte Multilayer Films Measured by Colloidal Probe AFM. Microsc. Res. Techniq. 2006, 69, 84-92.

(37) Landau, L. D.; Lifshitz, E. M. Theory of Elasticity; Pergamon Press Ltd: Oxford, 1959; pp 30-36.

(38) Köhler, K.; Shchukin, D. G.; Möhwald, H.; Sukhorukov, G. B. Thermal Behavior of Polyelectrolyte Multilayer Microcapsules. 1. The Effect of Odd and Even Layer Number. J. Phys. Chem. B 2005, 109, 18250-18259.

(39) Elsner, N.; Dubreuil, F.; Weinkamer, R.; Wasicek, M.; Fischer, F. D.; Fery, A. Mechanical Properties of Freestanding Polyelectrolyte Capsules: A Quantitative Approach Based on Shell Theory Characterization of Polymer Surfaces and Thin Films. Prog. Colloid Polym. Sci. 2006, 132, 117-123.

(40) Reissner, E. Stresses and Small Displacements of Shallow Spherical Shells. J. Math. Phys. 1946, 25, 80-85.

(41) Fery, A.; Weinkamer, R. Mechanical Properties of Microand Nanocapsules: Single-Capsule Measurements. Polymer 2007, 48, 7221-7235.

(42) Gregory, R. D.; Milac, T. I.; Wan, F. Y. M. A Thick Hollow Sphere Compressed by Equal and Opposite Concentrated Axial Loads: An Asymptotic Solution. SIAM J. Appl. Math. 1998, 59, 1080-1097.

(43) Mercadé-Prieto, R.; Thomas, C. R.; Zhang, Z. Mechanical Double Layer Model for Saccharomyces Cerevisiae Cell Wall. Eur. Biophys. J. 2013, D0I:10.1007/s00249-013-0909-x.

(44) McNamee, C. E.; Yamamoto, S.; Higashitani, K. Preparation and Characterization of Pure and Mixed Monolayers of Poly(ethylene Glycol) Brushes Chemically Adsorbed to Silica Surfaces. Langmuir 2007, 23, 4389-4399.

(45) Still, T.; Sainidou, R.; Retsch, M.; Jonas, U.; Spahn, P.; Hellmann, G. P.; Fytas, G. The "Music" of Core-Shell Spheres and Hollow Capsules: Influence of the Architecture on the Mechanical Properties at the Nanoscale. Nano Lett. 2008, 8, 3194-3199.

(46) Boudou, T.; Crouzier, T.; Auzely-Velty, R.; Glinel, K.; Picart, C. Internal Composition versus the Mechanical Properties of Polyelectrolyte Multilayer Films: The Influence of Chemical CrossLinking. Langmuir 2009, 25, 13809-13819.

(47) Glynos, E.; Koutsos, V.; McDicken, W. N.; Moran, C. M.; Pye, S. D.; Ross, J. A.; Sboros, V. Nanomechanics of Biocompatible Hollow Thin-Shell Polymer Microspheres. Langmuir 2009, 25, 7514-7522.

(48) Gao, C.; Donath, E.; Moya, S.; Dudnik, V.; Möhwald, H. Elasticity of Hollow Polyelectrolyte Capsules Prepared by the Layer-by-Layer Technique. Eur. Phys. J. E 2001, 5, 21-27.

(49) Nolte, A. J.; Rubner, M. F.; Cohen, R. E. Determining the Young's Modulus of Polyelectrolyte Multilayer Films via StressInduced Mechanical Buckling Instabilities. Macromolecules 2005, 38, 5367-5370.

(50) Bathe, K.-J.; Chapelle, D.; Lee, P.-S. A Shell Problem 'Highly Sensitive' to Thickness Changes. Int. J. Numer. Meth. Eng. 2003, 57, 1039-1052.

(51) Love, A. E. H. The Small Free Vibrations and Deformation of a Thin Elastic Shell. Philos. T. Roy. Soc. A 1888, 179, 491-546. 
Table of Contents entry.

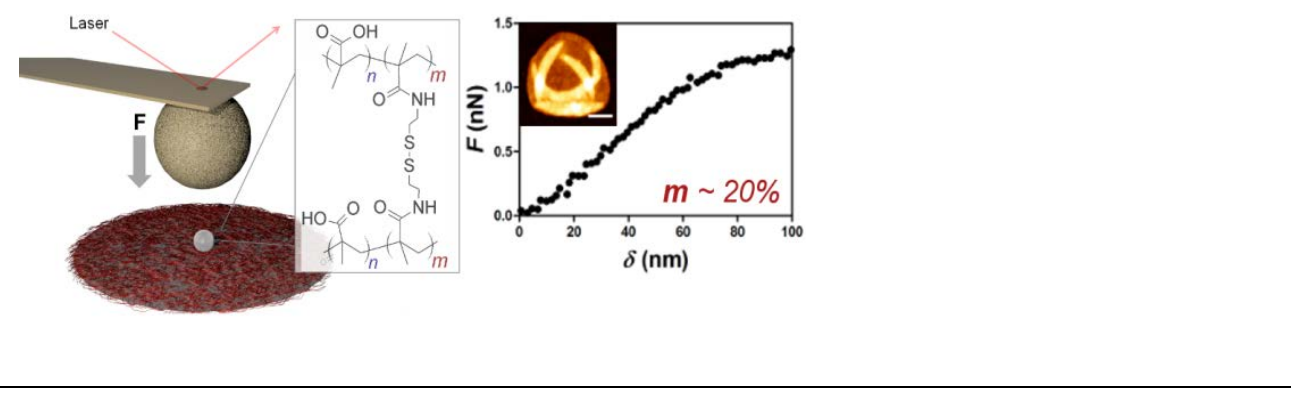

13 


\section{University Library}

\section{- M M I E E R VA A gateway to Melbourne's research publications}

Minerva Access is the Institutional Repository of The University of Melbourne

Author/s:

Best, JP;Neubauer, MP;Javed, S;Dam, HH;Fery, A;Caruso, F

Title:

Mechanics of pH-Responsive Hydrogel Capsules

Date:

2013-08-06

Citation:

Best, J. P., Neubauer, M. P., Javed, S., Dam, H. H., Fery, A. \& Caruso, F. (2013). Mechanics of pH-Responsive Hydrogel Capsules. LANGMUIR, 29 (31), pp.9814-9823. https:// doi.org/10.1021/la402111v.

Persistent Link:

http://hdl.handle.net/11343/123297 